\title{
Health information system for community-based multiple screening in Keelung, Taiwan (Keelung Community-based Integrated Screening No. 3)
}

\author{
Yueh-Hsia Chiu a,b, Li-Sheng Chen ${ }^{\text {a }}$, Chang-Chuan Chan ${ }^{c}$, Der-Ming Liou ${ }^{a}$, \\ Shiao-Chi Wu ${ }^{d}$, Hsu-Sung Kuo ${ }^{a}$, Hong-Jen Chang ${ }^{e}$, Tony Hsiu-Hsi Chen ${ }^{f, *}$
}

\author{
a Institute of Public Health and Institute of Health Informatics and Decision Making, National Yang-Ming \\ University, Taipei, Taiwan \\ ${ }^{\mathrm{b}}$ Health Bureau of Keelung City, Keelung City, Taiwan \\ c Institute of Occupational Medicine and Industrial Hygiene, College of Public Health, National Taiwan \\ University, Taipei, Taiwan \\ d Institute of Health and Welfare Policy, National Yang-Ming University, Taipei, Taiwan \\ e Department of Health (DOH), Executive Yuan, Taiwan \\ f Institute of Preventive Medicine, College of Public Health, National Taiwan University, Room 207, \\ 2F No. 19, XuZhou Road, Taipei 100, Taiwan
}

Received 29 April 2004; received in revised form 12 July 2005; accepted 13 July 2005

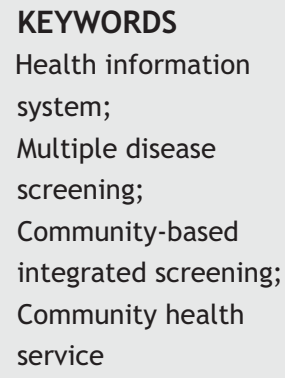

\begin{abstract}
Summary
Background: Community-based multiple screening for common cancers and chronic diseases has increasingly gained attention. However, as infrastructure and evaluation system are more diversified and complicated compared with single screening, the development of a novel health information system is paramount.

Methods: The main goal of our health information system was to support the multiple screening program not only from technical aspect but also from a broad range of perspectives including quality assurance system, organized features appertaining to screening, economic evaluation (cost-effectiveness or cost-utility analysis), epidemiological applications, behavior risk factor surveillance system, and social impact due to the introduction of the Keelung Community-Based Integrated Screening (KCIS) program.

Results: Health information system was designed and programmed on the basis of the demand derived from KCIS within which five cancers and three chronic diseases were included. In addition to the detailed description of infrastructure and process, design, relevant database and security involved in health information system, an
\end{abstract}

\footnotetext{
* Corresponding author. Tel.: +886 2 23587620; fax: +886 223587707
}

E-mail address: stony@episerv.cph.ntu.edu.tw (T.H.-H. Chen). 
innovative and extensive evaluation system in accordance with the main goals was included in our health information system.

Conclusion:: Our information system proposed several aspects regarding organized screening system that has never been addressed in computerized system supporting for single screening. These included quality assurance system, organization features, co-morbidity profiles, epidemiological applications and social and economic considerations.

๑) 2005 Elsevier Ireland Ltd. All rights reserved.

\section{Introduction}

Mass screening for reducing mortality from cancers or chronic diseases has been demonstrated in earlier studies, including breast cancer screening with mammography $[1,2]$, colorectal cancer screening with fecal occult blood test (FOBT) $[3,4]$, cervical cancer screening with Pap smear test [5], and early detection of type 2 diabetes and hypertension [6], respectively.

As each single screening has been proven effective, community-based multiple screening for the inclusion of these cancers and chronic diseases has increasingly gained attention [7]. However, the infrastructure and evaluation system required for a multiple screening program are more complicated compared to those for single screening, i.e. cervical cancer screening proposed by Marrett et al. [8]. The complexity can be viewed from several angles. First, the appropriate age ranges for the various diseases included in the multiple screening program may vary. For instance, an age range of 50-79 years may be recommended for colorectal cancer screening whereas 30-79 years for cervical cancer screening. Second, the inter-screening interval varies with disease type as well as with age group. In breast cancer screening, an annual screening regime is suggested for young women [9] but a triennial screening program is mostly used for old women [10]. Furthermore, the inter-screening interval may also vary with different diseases. An annual FOBT is recommended for average-risk people aged 50 years or more [3] but a 3 yearly screening regime is suggested for breast cancer screening with mammography for the same woman. The more diseases under consideration the more complicated the information system will be with respect to the heterogeneous inter-screening intervals. Third, as organized screening programs are characterized by an out-reaching service as a first step and confirmatory diagnosis and referral of abnormal cases at a second stage, concern is raised over the multiplication of health manpower required to handle the referrals and confirmatory diagnoses in the same subject given two or more abnormal findings.
Fourth, clinical surveillance of high-risk group is also complex when the number of diseases under consideration increases. Clinical surveillance for patients diagnosed as colorectal adenoma together with cervical intraepithelial neoplasia $(\mathrm{CIN}) 3$ is more complicated than when one only diagnosis is involved. Fifth, clinical management of asymptomatic cancers or chronic diseases requires the integration of inter-disciplinary specialty. Sixth, the procedures required in multiple screening are also more sophisticated with respect to the identification and invitation of eligible subjects, computer registration of attendants at on-site screening, and linkage between primary data and archival data. The preparation of archival data before screening should consist of retrieval from several registrybased resources, including the national health insurance, the cancer registry, the Pap smear registry and the mortality registry. Primary data from questionnaires or biological measures are also comprehensive as to cover as much issues as possible related to the diseases considered for screening. Finally, the evaluation of efficacy and effectiveness of a multiple screening program may also be different from the evaluation of a single screening regime.

The main goals of our health information system for multiple screening were: (1) to develop a quality assurance system for screening cancers as well as chronic diseases; (2) to assess how multiple screening can be organized in terms of health manpower, mode of early detection of cancers or chronic diseases (out-reach/opportunistic), age ranges and inter-screening intervals, the referral and confirmatory diagnosis system, and the clinical surveillance system; (3) to develop an outcome evaluation system, including effectiveness or economic evaluation (cost-effectiveness or cost-utility analysis); (4) to do epidemiological evaluation such as co-morbidity profiles, and familial aggregation or genetic contribution; (5) to set up a behavior risk factor surveillance system and (6) to evaluate social impact due to the introduction of the Keelung Community-Based Integrated Screening (KCIS) program. 


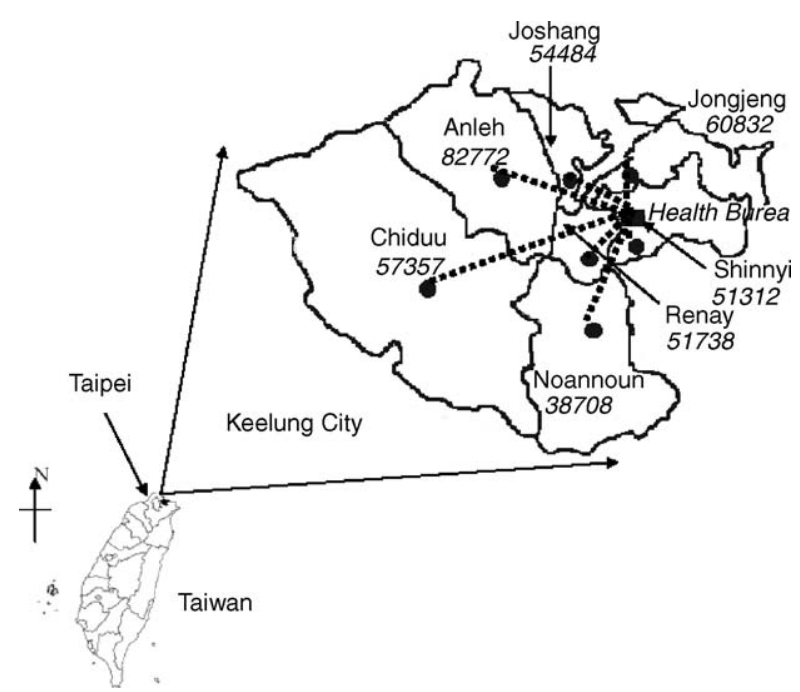

Fig. 1 The distribution of geographical area and network backbone of Keelung City.

The rest of this paper was organized as follows. Section 2 refers to background of KCIS. Section 3 delineates infrastructure, database, administration support and security. Section 4 gives the detailed description of evaluation system from different aspects. Section 5 gives the discussion of this paper.

\section{Background}

\subsection{Architecture of health information network of Keelung City}

Keelung City is located at the northernmost Taiwan and close to the capital, Taipei. There are seven administration districts (Joshang, Jongjeng, Shinnyi, Renay, Noannoun, Anleh and Chiduu) in Keelung as shown in Fig. 1. The total area covered is 132.76 square kilometers $\left(\mathrm{km}^{2}\right)$. Each district has one local health service center, the basic administrative unit responsible for the execution of the multiple screening program in Keelung (see below). These seven health centers were ramified from the Health Bureau of Keelung City, which is the headquarter of health policy and administration. To exchange and share information, the seven health centers are connected with the Health Bureau by leased line or ADSL underpinning National Health Information Network Service Center (NIH-SC) set up in 1995 (Fig. 1) by Ministry of Health in Taiwan.

\subsection{Multiple screening model and screened population}

In Taiwan, a nationwide policy of cervical cancer screening and surveillance system of Pap smear has been established in 1996. Selective screenings for breast cancer, oral cancer and colorectal cancer have been launched since 1997. The prevalence of chronic diseases, such as type 2 diabetes, hypertension and cardiovascular disease increased over the years. To enhance the feasibility and acceptability of each individual screening, a multiple screening model for the Keelung community, named as Keelung Community-Based Integrated Screening, was developed between 1999 and 2001 to integrate screening for five types of cancers (cervical cancer, breast cancer, colorectal cancer, oral cancer and liver cancer) and three types of chronic diseases (hypertension, hyperlipidemia and type 2 diabetes). The target population invited to screen consisted of 217,895 residents aged 30-79 years (Table 1). The detailed procedures and criteria of disease screening have been described in full elsewhere [7]. This model has been continuously applied to cover $28.4 \%$ of the total target population, amounting to 61,869 attendants until the end of 2003 (Table 1).

\section{Materials and methods}

\subsection{Infrastructure and procedure}

Supporting multiple screening processes with information technology requires workflow specifi-

Table 1 Target population, attendants and coverage rate of the underlying population

\begin{tabular}{|c|c|c|c|c|c|c|c|}
\hline \multirow[t]{2}{*}{ Age group } & \multicolumn{3}{|c|}{ Target population } & \multicolumn{3}{|c|}{ Attendants } & \multirow[t]{2}{*}{ Coverage rate (\%) } \\
\hline & Male & Female & Total & Male & Female & Total & \\
\hline $30-39$ & 36255 & 34468 & 70723 & 4334 & 8935 & 13269 & 18.8 \\
\hline $40-49$ & 33371 & 32371 & 65742 & 6067 & 10855 & 16922 & 25.7 \\
\hline $50-59$ & 17440 & 18016 & 35456 & 4395 & 7944 & 12339 & 34.8 \\
\hline $60-69$ & 12554 & 13646 & 26200 & 4421 & 7633 & 11154 & 42.6 \\
\hline $70-79$ & 11077 & 8697 & 19774 & 4145 & 4040 & 8185 & 41.4 \\
\hline Total & 110697 & 107198 & 217895 & 23362 & 38507 & 61869 & 28.4 \\
\hline
\end{tabular}


cation, such as identification of tasks, procedures of screening, input and output information and information management. The infrastructure and procedures of the CIS information system were divided into three phases, pre-screening phase, screening phase and post-screening phase.

The process in the pre-screening phase included data linkage and filtering. Through the linkage process, subjects ascertained as death through mortality registry were excluded from invitation list. The cancer registry was used to determine whether the subject had been diagnosed as cancer before screening. Subjects with previously certain diagnosed cancer were not invited to attend screening for this specific cancer. However, she/he was still eligible to attend other screening activities. We obtained data on utilization of medical care and history of chronic disease, particularly including type 2 diabetes, hypertension and cardiovascular disease from claim data from the national health insurance. Pap smear screening database was used to assess whether women who attended screening had been undergone Pap smears within the recent 3 years because the three-yearly screening regime has been applied to the KCIS program.

After this filtering process, we produced for each household unit in each community parish an invita- tion list with known information including history of Pap smear, and history of chronic disease, history of other screening items done fore. Since Pap smear screening is a national program, the corresponding database has been centralized. Other screening projects, not included in a national program, were obtained from our community-based screening program. Thus, primary data generated from the previous post-screening phase were used to renew the history of each disease.

The screening phase, consisted of nine items (Fig. 2). The first item was registration. The output of the pre-screening phase regarding selected and classified invitation list was an input to the screening phase. Subjects on the list were invited to screening by a telephone call done by a public health nurse. Attendants in the KCIS were registered. According to the classification list in the first phase, participants were informed about how many diseases he/she should be screened on this occasion. In the registration process, a document related to insurance claims was filled out and demographic information was updated if needed. The item after registration was blood drawing. Usually blood sample screening was done for type 2 diabetes (fasting blood sugar), hyperlipidemia (cholesterol, triglyceride, high density lipid (HDL), low

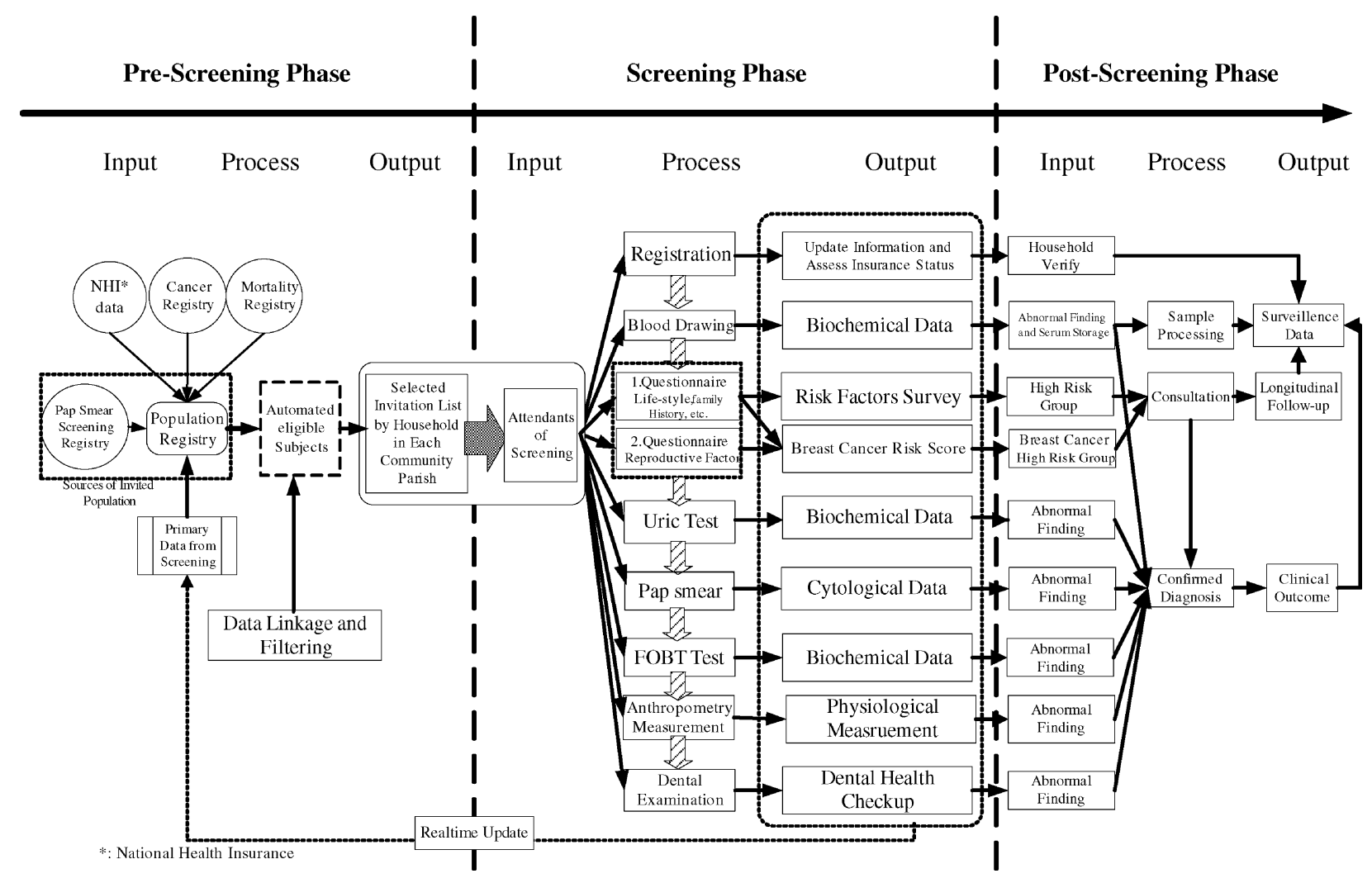

Fig. 2 The infrastructure and workflow of building-up information system for multiple screening. 
density lipid (LDL)), liver cancer first-stage screening (HBsAg, Anti-HCV, $\alpha$-fetoprotein (AFP), alanine aminotransferase (ALT) and aspartate aminotransferase (AST)), and health check-up for uric acid, BUN, creatinine. The sequence in which the other screening items were performed was flexible and varied by location and occasion. In the example in Fig. 2, the third item is a questionnaire for health risk appraisal. The questionnaire covers behavior risk factors, family history and personal disease history, and female reproductive factors. Highrisk subjects of breast cancer were determined by reproductive factors. The fifth was a urine test to get biochemical data on urine protein, sugar, $\mathrm{pH}$ and so on. The sixth was Pap smear examination for early detection of cervical neoplasm. The seventh was related to the FOBT test for colorectal cancer screening. The eighth item consists of anthropometrical measurements, such as height, weight, waist-to-hip ratio, blood pressure. The ninth was dental examination for the identification of oral pre-malignancy and oral cancer. Each item results in a set of data that is stored in a specific database.

\subsection{Database of CIS}

Table 2 shows the relevant databases involved in the multiple screening program. They are classified into two types, secondary and primary data. Primary data are generated during the screening; secondary data are obtained from elsewhere and used as input for the screening program. Secondary data consist of population registry, Pap smear registry, cancer registry, claim data on national health insurance and mortality data. Primary data include questionnaire of behavior risk factor, personal disease history and family history, biochemical data obtained from blood sample screening and followup system (referral and confirmatory diagnosis). Table 2 also shows type of data, source of database, character and frequency of renewal. It should be noted that, for mortality, we updated information weekly on the basis of local mortality data. To capture the migrants who have been dead outside Keelung, national mortality registry was used to update information annually.

\subsection{Administration support}

Our health information system also provided each one attending the screening the option make their appointment through internet or through the telephone registration system. Time schedules for the appointment were posted on the website for reference 1 month before each screening activity.

A temporary server was set up for this community out-reaching services. The main server was installed at the headquarters of Keelung Health Bureau. Basic information was downloaded from the main server to temporary server as the first step of registration and to provide the report regarding the result of check-up in the previous year. After out-reaching services, new lists of registration were generated and uploaded to the main server for updating population-based database (Fig. 3).

Our information system supported follow-up and referral systems and confirmatory diagnoses for the patients for which abnormal findings were found during the screening. The system also produced electronic reports on the results of biochemical test, follow-up, confirmatory diagnosis, health consultation and health education or primary prevention of relevant disease. Real-time and flexible

Table 2 Dataset used in community-based multiple screening

\begin{tabular}{|c|c|c|c|}
\hline Items & Source & Character & Renewal \\
\hline Population registry & Bureau of Census & Secondary data & Annually \\
\hline Pap smear screening registry & Ministry of Health & Secondary data & Annually \\
\hline Cancer registry & Ministry of Health & Secondary data & Annually \\
\hline National health insurance & $\begin{array}{l}\text { Bureau of National } \\
\text { Health Insurance }\end{array}$ & Claim data & Annually \\
\hline Mortality registry & Ministry of Health & Secondary data & $\begin{array}{l}\text { Annual update from national } \\
\text { mortality and weekly update } \\
\text { from local mortality data }\end{array}$ \\
\hline Questionnaire & $\begin{array}{l}\text { Community-based } \\
\text { Risk Factor Survey }\end{array}$ & Primary data & Annually \\
\hline Biochemical data & $\begin{array}{l}\text { Community-based } \\
\text { Screening }\end{array}$ & Primary data & Depends on disease \\
\hline Follow-up system & Referral Hospital & Primary data & Depends on disease \\
\hline
\end{tabular}




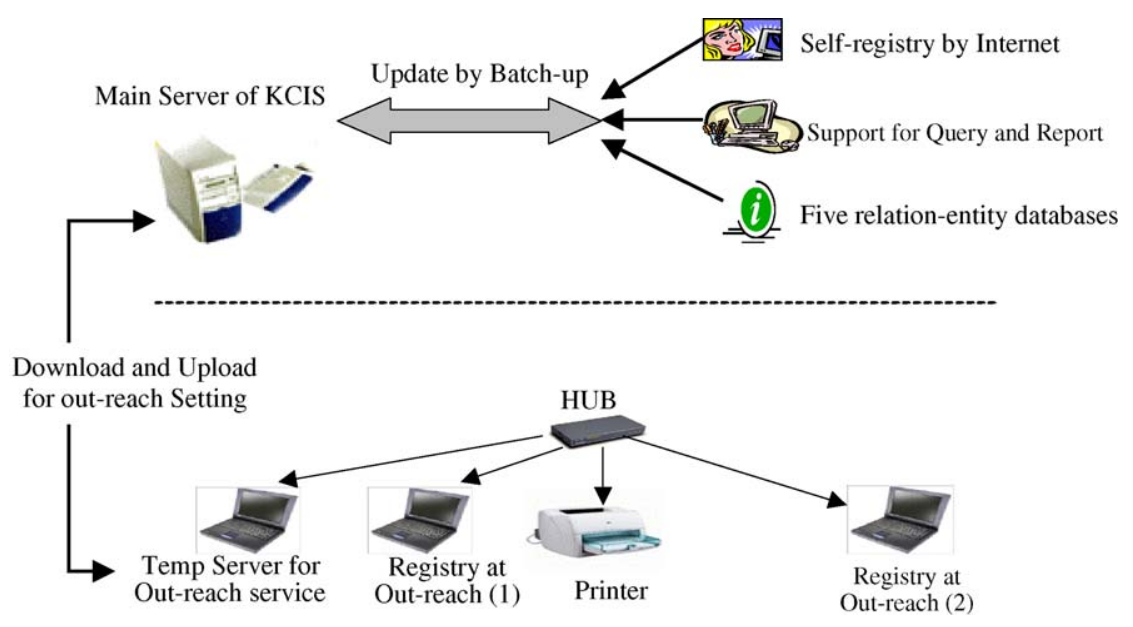

Fig. 3 Ambulatory support setting for community out-reach services.

criteria query functions were also embedded in system as required by the staff.

\subsection{Security}

For each screenee, an individual informed consent was given while he/she attended the on-site screening. Individual data on occurrence of cancer, death and medical utilization during the follow-up period were updated by cancer registry, mortality registry data and claim data from the national health insurance, respectively. Security control was implemented by including only the identification number without any further description of characteristics of patient such as name and correspondence address in the formal transactions between two or three administrations.

To protect confidentiality and security of personal health information, the strictest standard procedures and a special privacy policy statement was proposed and audited. Users of the information system are first informed about this statement before they login with a password. Signing this statement by the staff was required. Users are given access to functionalities dependent on their roles in the screening process, e.g. upload of biochemical test data, download of ambulatory database for registration and update of follow-up information. Firewall, fixed access IP and password verification were incorporated to guard against infringement of the database.

\section{Evaluation system}

The evaluation system of the KCIS included several aspects that are delineated in Table 3.

\subsection{Quality assurance system}

In a multiple screening program, it is paramount to link together a series of registration data in the pre-screening phase (see Fig. 2). Doing so not only yielded invitation lists for screening but also offered an opportunity to build up a data quality assurance system in the multiple screening program.

There are five dimensions related to the quality assurance of the KCIS program (Table 3). Selected results for some indicators based on the KCIS study will be presented. First, the linkage of screening data with the claim data from the national health insurance can assess the validity of self-reported chronic diseases such as type 2 diabetes and hypertension. The misclassification of self-reported diseases can be expressed by sensitivity and specificity.

Second, the linkage of primary data from the KCIS program with national health insurance and cancer registry data enables us to assess the comprehensiveness timeliness of reporting to the cancer registry for the five neoplastic diseases covered by our multiple screening program. Such an analysis shows that the proportions for colorectal cancer and Hepatocellular carcinoma reported to the national cancer registry until the end of 2003 among screen-detected cases in the same period were 74.4 and $88.4 \%$, respectively (Table 3 ).

Third, the linkage of attendants who were diagnosed as free of cancer at screening with the national cancer registry can ascertain occurrence of interval cancers (cancers diagnosed between screens), a crucial indicator for evaluation of program sensitivity and inter-screening interval in population-based cancer screening (see below) [8]. The higher the interval cancer rate the poorer the 


\begin{tabular}{|c|c|c|}
\hline Components & Methods/data sources & $\begin{array}{l}\text { Indicators/selected results from the KCIS } \\
\text { program/comments }\end{array}$ \\
\hline $\begin{array}{l}\text { 1. Quality assurance } \\
\text { (1.1) Consistency }\end{array}$ & $\begin{array}{l}\text { Self-reported disease from KCIS data vs. claim } \\
\text { data from national health insurance (NHI) }\end{array}$ & $\begin{array}{l}\text { Sensitivity and specificity for Self-reported disease (yes or } \\
\text { no) cross-tabulated by data from NHI } \\
\text { i.e. Type } 2 \text { diabetes: Sen, } 76.37 \% \text {; Spe, } 97.52 \%\end{array}$ \\
\hline $\begin{array}{l}\text { (1.2) Comprehensiveness or delay report } \\
\text { of national cancer register }\end{array}$ & $\begin{array}{l}\text { Proportion of cancers reported to national } \\
\text { cancer registry among screen-detected cases } \\
\text { at of specific time }\end{array}$ & $\begin{array}{l}\text { Cases reported to cancer registry/cases from the KCIS } \\
\text { program until } 2003 \\
\text { i.e. Colorectal cancer: } 74.42 \%(32 / 43) \\
\text { i.e. Hepatoma: } 88.41 \%(122 / 138)\end{array}$ \\
\hline $\begin{array}{l}\text { (1.3) Interval cancer rate } \\
\text { (1.4) Cumulative survival rate }\end{array}$ & $\begin{array}{l}\text { Interval cases found by linking normal subjects } \\
\text { at screen cancers with national cancer registry } \\
\text { Number of death from screen-detected cases } \\
\text { or clinically-detected cases by the linkage of } \\
\text { the KCIS data with national cancer registry }\end{array}$ & $\begin{array}{l}\text { Program sensitivity }=[1-(\text { interval cancer rate/the } \\
\text { expected incidence rate) }] \times 100 \% \text {, i.e. CRC: } 85 \% \\
1,5 \text {, or } 10 \text { year cumulative survival rate }\end{array}$ \\
\hline \multirow[t]{4}{*}{ (1.5) Data quality of questionnaire } & $\begin{array}{l}\text { (a) Any key variables of questionnaire were } \\
\text { missing }\end{array}$ & $\begin{array}{l}\text { (a) Missing records/total records } \\
\text { i.e. Missing rate }(2000-2002): 2.27 \% \rightarrow 0.00 \%\end{array}$ \\
\hline & (b) Duplicate cases within a year & $\begin{array}{l}\text { (b) Duplicate attendants/total attendants } \\
\text { i.e. Duplicating rate }(2000-2002): 3.97 \% \rightarrow 0.02 \%\end{array}$ \\
\hline & (c) Data logical checking & $\begin{array}{l}\text { (c) Error records/total records } \\
\text { i.e. Inaccurate rate }(2000-2002): 0.86 \% \rightarrow 0.00 \%\end{array}$ \\
\hline & (d) Household coverage rate & $\begin{array}{l}\text { (d) Households of KCIS/total households } \\
\text { i.e. } 35.8 \%(48166 / 134607)\end{array}$ \\
\hline $\begin{array}{l}\text { 2. Organization } \\
\text { (2.1) Coverage rate }\end{array}$ & $\begin{array}{l}\text { Proportion of population served with the KCIS } \\
\text { project }\end{array}$ & $\begin{array}{l}\text { Total attendants/total population } \\
\text { i.e. age } 30-79(2000-2002): 19.5 \% \rightarrow 28.4 \%\end{array}$ \\
\hline (2.2) Outreach/opportunistic screening & $\begin{array}{l}\text { Linkage of the KCIS data with claim data from } \\
\mathrm{NHI} \text { for adult health check-up }\end{array}$ & $\begin{array}{l}\text { Attendants of outreach since from the KCIS/attendant } \\
\text { from opportunistic visit, i.e. adult health checkup: } 43 \% \text {; } \\
\text { KCIS attendants: } 57 \%\end{array}$ \\
\hline $\begin{array}{l}\text { (2.3) Health manpower involved in on- } \\
\text { site screening }\end{array}$ & $\begin{array}{l}\text { Number and composition (volunteer social } \\
\text { worker vs. professional health manpower) of } \\
\text { health manpower }\end{array}$ & $\begin{array}{l}\text { (a) Average served attendants per manpower: } 2.2 \\
(2000) \rightarrow 4.4 \text { (2003) } \\
\text { (b) The proportion of volunteer social worker on } \\
\text { professional health manpower: } 25.4 \%(2000) \rightarrow 32.1 \%(2003)\end{array}$ \\
\hline
\end{tabular}




\begin{tabular}{|c|c|c|}
\hline Components & Methods/data sources & $\begin{array}{l}\text { Indicators/selected results from the KCIS } \\
\text { program/comments }\end{array}$ \\
\hline (2.4) Referral and confirmatory diagnosis & $\begin{array}{l}\text { Clinical capacity for referral and confirmatory } \\
\text { diagnosis }\end{array}$ & $\begin{array}{l}\text { (a) Mean waiting time for referral and confirmatory } \\
\text { diagnosis } \\
\text { (b) Times of referral }\end{array}$ \\
\hline $\begin{array}{l}\text { (2.5) Age ranges and inter-screening } \\
\text { interval }\end{array}$ & Depending on diseases & $\begin{array}{l}\text { Colorectal Cancer: } 50-79 \text {, annually } \\
\text { Cervical cancer: } 30-79 \text {, every } 3 \text { years }\end{array}$ \\
\hline (2.6) Clinical surveillance & Depend on characteristic of screening finding & $\begin{array}{l}\text { Pre-cancer lesion: colorectal cancer } \\
<1 \mathrm{~cm} \text { adenoma: } 3-6 \text { years after the initial polypectomy } \\
\geq 1 \mathrm{~cm} \text { adenoma: within } 3 \text { years after initial polypectomy }\end{array}$ \\
\hline $\begin{array}{l}\text { 3. Basic findings } \\
\text { (3.1) Attendance rate }\end{array}$ & $\begin{array}{l}\text { Attendance rate of before and post } \mathrm{KCIS} \\
\text { projects }\end{array}$ & $\begin{array}{l}\text { Attendants/number of Invitation } \\
\text { i.e. Cervical cancer: Before: } 55.5 \% \rightarrow \text { Post: } 80.5 \%\end{array}$ \\
\hline (3.2) Abnormal rate & Abnormal rates of screening test & $\begin{array}{l}\text { Positive of test/total attendants } \\
\text { i.e. FOBT positive rate (2001-2003): } 4.1 \%, 4.5 \%, 3.7 \%\end{array}$ \\
\hline (3.3) Referral performance & Calculated data by referral part of database & $\begin{array}{l}\text { Referral cases/abnormal finding }(2000-2002) \\
\text { i.e. Colonoscopy for CRC: } 67.13 \% \rightarrow 73.97 \% \\
\text { i.e. Ultrasonography for HCC: } 77.37 \% \rightarrow 88.52 \%\end{array}$ \\
\hline (3.4) Biopsy rate & Confirmatory biopsy of abnormal findings & $\begin{array}{l}\text { Biopsy cases/abnormal cases } \\
\text { i.e. Breast cancer: } 2.87 \%\end{array}$ \\
\hline \multicolumn{3}{|l|}{ 4. Effectiveness } \\
\hline (4.1) Prevalence/incidence ratio & $\begin{array}{l}\text { Prevalence rate }(\mathrm{P}) \text { from the } K C I S \text { and } \\
\text { incidence (I) from national cancer registry }\end{array}$ & $\begin{array}{l}\text { Prevalence rate from screen/incidence from cancer } \\
\text { registry. Average duration of pre-clinical phase (in year), } \\
\text { i.e. } \text { CRC: } 3.7 \text { year }\left(1.52 \times 10^{-3} / 4.1 \times 10^{-6}\right)\end{array}$ \\
\hline (4.2) I/E ratio & $\begin{array}{l}\text { See the point (1.3) of quality assurance } \\
\text { mentioned above }\end{array}$ & See above \\
\hline $\begin{array}{l}\text { (4.3) Proportion or cumulative incidence } \\
\text { rate of advanced cancers or }\end{array}$ & $\begin{array}{l}\text { Proportion or cumulative incidence rate of } \\
\text { large tumor, lymph nodes, and poor } \\
\text { differentiation }\end{array}$ & $\begin{array}{l}\text { Number of tumor characteristic/cancer cases } \\
\text { i.e. Dukes stages of CRC: Dukes A: } 13(39.4 \%) \text {, Dukes B:13 } \\
(39 . \overline{4 \%}) \text {, Dukes C: } 6(18.2 \%) \text {, Dukes D: } 1(3.0 \%)\end{array}$ \\
\hline $\begin{array}{l}\text { (4.4) Abnormal outcomes of relative } \\
\text { chronic disease }\end{array}$ & $\begin{array}{l}\text { Proportion of abnormal outcomes of chronic } \\
\text { diseases }\end{array}$ & $\begin{array}{l}\text { Hyperlipidemia: total cholesterol } \geq 240 \mathrm{ng} / \mathrm{dl} / \text { total } \\
\text { hyperlipidemia: } 12.7 \%\end{array}$ \\
\hline
\end{tabular}


(4.5) Long-term mortality

5. Co-morbidity profiles

6. Behavior risk factor surveillance

7. Decision/economic evaluation

(7.1) Cost-effectiveness analysis

(7.2) Cost-utility analysis

8. Epidemiological profiles

\section{Social aspect}

\section{(9.1) Cue to attending KCIS}

\section{(9.2) Satisfaction toward the KCIS}

(9.3) Interaction with local primary care or public health system
Linkage data to ascertain date of death and cause of death

(a) Empirical data

(b) Project: Monte Carlo Computer simulation

Attendants have more than one disease simultaneously

Life-style factors and biochemical data from the KCIS program

Markov decision analysis (see Fig. 4) of cost and effectiveness for the following comparisons

(1) Multiple/single screening

(2) Single screening/none

Linkage to national cancer registry to ascertain incident tumors and active follow-up of chronic disease

\section{Survey by random sampling}

Survey by random sampling from attendants in the KCIS

Participation and engagement from primary

care unit or specialist
Relative mortality rate for screened vs. unscreened

Attendants of $\geq 2$ types of disease/total attendants i.e. chronic disease: two: $20.7 \%$, three: $7.0 \%$ and four: $0.9 \%$

Monitor the changes of these factors and biochemical markers through repeated screens in the KCIS program

Incremental cost-effectiveness or utility ratio expressed by cost per life year gained or cost per quality-adjusted life year gained

(a) A series of longitudinal outcomes for elucidating causal relationships between baseline covariates and cancer or chronic disease through case-cohort or nested case-control study

(b) Family-based epidemiological design for family aggregation or genetic contribution

Heard about the KCIS/total survey cases $49.3 \%$ $(2000) \rightarrow 65.6 \%$ (2003)

Number of attendants toward satisfaction/Total samples: $81.4 \%(2000) \rightarrow 93.9 \%$ (2003)

Proportion of GP or social works included in the KCIS

involved in the $\mathrm{KCIS}$ program 
performance of the screening regime. The interval cancer rate as a percentage of the underlying incidence rate for colorectal cancer was $15 \%$, which, in turn, yielded $85 \%$ program sensitivity.

Fourth, the linkage of primary data on screendetected cases from the KCIS program and clinically-detected cases ascertained from the cancer registry and the national health insurance data with mortality allows us to evaluate the benefit of screening by comparing cumulative survival rates between early screen-detected cases and clinicallydetected cases.

Fifth, our information system also evaluated the aspect of completeness and accuracy of data on questionnaires. The missing rates decreased from 2.27 to $0.00 \%$ through the period from 1999 to 2002 . The duplicate rates decreased from $3.97 \%$ in 1999 to $0.02 \%$ in 2002 . Rate of inaccuracy decreased from $0.86 \%$ in 1999 to $0.00 \%$ in 2002 .

\subsection{Organizational aspects}

Evaluation of the KCIS program with respect to the organizational aspects includes coverage rate, outreach/opportunistic proportion, health manpower at on-site screening and referral and confirmatory diagnosis. Table 2 shows that the coverage rate increased from $19.5 \%$ in 2000 to $28.4 \%$ in 2002 .

Our national health insurance system fully subsidizes adult health check-up for chronic diseases such as type 2 diabetes, hypertension, and hyperlipidemia regardless of whether it is performed by opportunistic visits to primary care clinics or hospitals or out-reach services provided by the $\mathrm{KCIS}$ program. Analysis of the data showed that $57 \%$ of those screened for chronic diseases were reached by our KCIS out-reach service program.

The number of attendants served per unit manpower increased from 2.2 in 2000 to 4.4 in 2003, demonstrating the efficiency of on-site multiple screening.

The system also allows to evaluate other organizational aspects, e.g. whether clinical capacity for referral and confirmatory diagnosis can be met using indicators such as waiting time for specific disease or total of diseases included in the KCIS program. Times of referral and confirmatory diagnosis for multiple diseases included in the KCIS program can be assessed as well. It can also be determined whether loss to follow-up in a multiple screening program is lower compared with single screening from other data sources.

\subsection{Basic outcomes}

These included attendance rate, positive results of screening, referral rate, biopsy rate, detection rate of asymptomatic neoplasm or non-neoplastic chronic diseases, program sensitivity and specificity, positive predictive value for yield of screening.

In the KCIS program, we used the Pap smear registry as a base to invite other family members to have the uptake of screening. Such a strategy is innovative and practical because women can play an important role in caring for the health status of other family members. The empirical result from a previous study showed that the attendance rate for cervical cancer screening has been enhanced from 55 to $80 \%$ after the introduction of multiple screening [7]. Our health information system can also evaluate how women in the same family can affect the uptake of screening for other family members and how attendance rates vary with diseases included in the KCIS program.

Regarding referral rate, our information system can also assess whether the referral rate in the multiple screening program increased over time. For colorectal cancer screening, it increased from $67 \%$ in 2000 to $74 \%$ in 2002 . The rates for liver cancer screening increased from $77 \%$ in 2000 to $89 \%$ in 2002. The system can also check whether and why the referral rate varies with disease. For the yield of screening, our information system also assesses whether the multiple screening program can detect a higher proportion of asymptomatic diseases compared with single screening. The previous studies showed our multiple screening program can yield 16 per 1000 of asymptomatic neoplastic or nonneoplastic chronic diseases [7]. Other outcomes such as abnormal rates and biopsy rates are also listed in Table 3.

\subsection{Effectiveness of screening}

The assessments of the effectiveness of screening include short-term and long-term indicators. The former consist of prevalence/incidence (P/I) ratio, the incidence rate of interval cancer as a percentage of the expected incidence rate from the underlying population (I/E ratio), proportion or cumulative incidence rate of advanced cancer such as tumor larger than $2 \mathrm{~cm}$ or nodes positive. The higher $\mathrm{P} / \mathrm{I}$ ratio, the lower I/E ratio, and the lower the proportion or the cumulative incidence rate of advanced cancers, the better the benefit of screening achieved. For colorectal cancer we estimated 3.7 years of average duration of pre-clinical phase using $\mathrm{P} / \mathrm{I}$ ratio (Table 3 ), $15 \%$ of I/E ratio and $80 \%$ of early CRC (Dukes' A + Dukes' B).

The primary long-term outcome is mainly based on mortality reduction from specific causes of the underlying diseases that are covered in the KCIS 
program. However, since mortality or incidence needs long-term follow-up, a computer simulation system for predicting long-term mortality reduction or incidence reduction will be included in our future information system.

\subsection{Co-morbidity profiles}

As multiple disease screening may find multiple asymptomatic cases, our evaluation system also included the estimation of co-morbidity of diseases in each individual. The proportions of attendants with two, three and four chronic diseases are listed in Table 3. The co-morbidity of cancers and chronic diseases are also evaluated in the KCIS program.

\subsection{Behavior risk factor surveillance}

The KCIS program provides a platform for bridging screening with primary education, particularly pertaining to a health promotion program for prevention of chronic diseases. Repeated biochemical data together with life-style factors obtained from questionnaires in our KCIS program offer an opportunity to monitor changes in behavior risk factors, including the cessation rate of smoking and the changes of blood pressure, blood lipids, fasting blood level, dietary habits and status of physical activity after the introduction of a series of primary education programs, by comparing the mean or median for each specific biochemical variable before and after intervention.

\subsection{Decision/economic evaluation}

The novel idea of our multiple screening program is that the effectiveness of mortality reduction in multiple disease screening should represent early detection of multiple asymptomatic neoplasm or non-neoplastic chronic disease rather than only single disease. Markov decision analysis is applied to estimating multiple benefits for early detection of two non-neoplastic chronic diseases (including asymptomatic type 2 diabetes, and hypertension) and four common cancers (cervical neoplasm, oral neoplasm, breast cancer and colorectal neoplasm). The decision tree was delineated in Fig. 4. Three decisions were considered and represented as three branches after the decision node. After each decision, Markov node was shown to exhibit the repeated property for a longitudinal course. Each Markov node was followed by states of the six diseases of interest. The progression of the six diseases was determined by screening policy and its natural history.

This decision framework enables our health information system to perform economic evaluation of the KCIS program, particularly in comparison with single screening. Types of economic evaluation included are cost-effectiveness and costutility analysis. In cost-effectiveness analysis, we

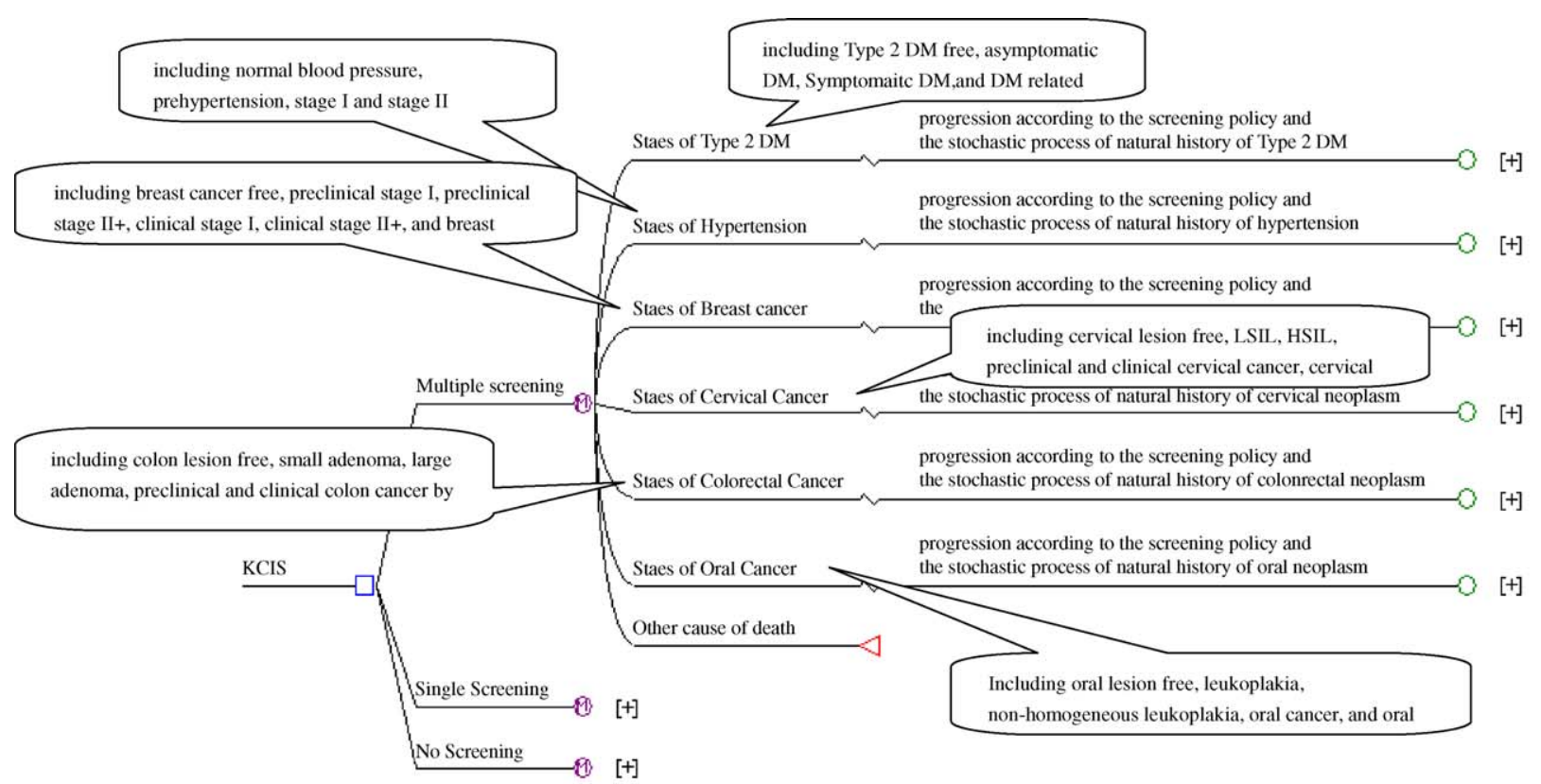

Fig. 4 Decision framework of multiple screening and single screening. 
compared the following counterparts of screening regimes: multiple versus single screening or single versus non-screening program by using incremental cost-effectiveness ratio, which aims to estimate incremental cost incurred for the KCIS program against single screening or single screening against non-screening program to gain an additional life year. A hypothetic cohort with similar features in distribution of age and gender of Keelung community was simulated. Underpinning the Markov decision framework mentioned above (see Fig. 4), people in the cohort were randomly assigned into the three arms, multiple screening, single screening and non-screening. After 6 year screening period and 10 year follow-up, multiple screening with $100 \%$ compliance rate yielded incremental cost of US\$ 6002 per life year gained compared to no screening performed. For single screening with $100 \%$ compliance rate, the corresponding figure was US\$ 8829 per life year gained. In cost-utility analysis, we translated life years into quality-adjusted life years (QALYs) by the incorporation of the utility of each health state to calculate incremental cost-utility ratio.

\subsection{Epidemiological profiles}

The follow-up of normal subjects in the KCIS program will yield a series of longitudinal incident cases regarding chronic diseases or cancers rather than single ones, which not only provides the chance of elucidating the associations between different outcomes but also throw light on causal relationships between baseline or time-dependent covariates and cancers or chronic diseases. In addition, household data from the KCIS program offers an opportunity to investigate family aggregation or genetic contribution using family-based epidemiological design.

\subsection{Social aspects}

From the social aspect, evaluation also included the assessment of satisfaction with the KCIS program. Each year approximately 200 people, randomly selected from the population registry in Keelung City are interviewed through the telephone to assess whether they have heard about the KCIS program. In this survey, the proportion of attendants who heard about the $\mathrm{KCIS}$ program increased from $49 \%$ in 2000 to $66 \%$ in 2003 . Of those who participated in the KCIS program, $82 \%$ in 2000 to $94 \%$ in 2003 were satisfied with the program. The social impact regarding interaction with local primary care or public health system was also included.

\section{Discussion}

The present study developed a novel health information system for the multiple screening program implemented in Keelung, Taiwan, which is called as Keelung Community-Based Integrated Screening program. Compared with the existing health information system for single screening such as cervical cancer screening [8], breast cancer screening $[11,12]$, and primary care for chronic disease $[13,14]$, the novel idea of our health information system for the KCIS program can be addressed from several dimensions, including: (1) the unification of a series of cancers and chronic diseases as a system; (2) quality assurance program with the incorporation of a series of registry-based or claim data; (3) household invitation system; (4) the consideration of heterogeneous organizational factors involved in multiple screening such as manpower, age ranges, referral and confirmatory diagnosis, clinical surveillance, and inter-screening interval; (5) economic evaluation of multiple versus single screening; (6) the collection of a series of longitudinal cancers and chronic diseases rather than single disease; (7) the integration of social aspect related to screening and (8) the combination of out-reach service and opportunistic screening.

The integration of cancers and chronic diseases as a union enables one to investigate comorbidity profiles and the association between cancer and chronic diseases. For example, those who were diagnosed as metabolic syndrome were at increased risk for colorectal neoplasm [15-17]. However, the incorporation of multiple diseases increased the complexity of organized screening such as age ranges and inter-screening intervals for difference cancers. Our KCIS system also supports clinical surveillance of high-risk group for colorectal cancer such as pre-cancerous lesion of colorectal adenoma or cervix and the deployment of health manpower involved at on-site screening, and referral and follow-up system for multiple diseases.

Another novel property of in our health information system is that our KCIS program used a Pap smear as a base to invite other family members by linkage with the population registry. Invitation is therefore based on the household unit what has been addressed barely in single screening for breast or cervical cancer. The incorporation of household information allows to evaluate the attendance rate not only on an individual basis but also based on the household unit. It should be noted that household invitation may not only increases coverage rate of screened population by inviting other family mem- 
bers who have been rarely involved in the uptake of screening but also reduce the cost and time for the public health nurse to generate an invitation list with repetition.

For quality assurance, the linkage of population registry data with cancer registry data or claim data from the national health insurance enables one to assess the validity of self-reported chronic diseases from questionnaires or biochemical tests or to assess comprehensiveness or delay of reporting to the national cancer registry. In addition, the linkage of the KCIS data with claim data on adult health check-up from the national health insurance can reveal whether out-reached services dominate opportunistic visits in routine primary care. When it comes to quality assurance for cancer screening, primary screened data can provide for pathological staging information on tumor size, nodes status and histological differentiation that may offset the weakness of being unable to collect staging information in the cancer registry system, one of limitations of quality assurance programs in some countries participating in the International Breast Cancer Screening Network (IBSN) [11,12]. Until now, the Taiwan cancer registry has not collected staging information.

Our information system has also strength in facilitating on-site screening activities. These include the determination of inter-screening intervals for all sorts of screening items according to different individual characteristics such as age and risk factors, the update of personal correspondence information such as number of cell phone, the results of previous screening for subjects attending the repeated screening, risk assessment of the presence of disease of interest given individual characteristics. The first aspect is crucial for multiple screening because it is difficult for attendants or personnel involved in periodical screening to apprehend whether he/she should undertake a certain screening test in this occasion without the aid of computer-based information system. The second point is related to the attendance rate of repeated screenings and the cost related to the invitation of subjects to attend the KCIS program. Due to the wide use of cellular phones in Taiwan, keeping the number of the mobile phone with the approval of participants up-to-date when they re-attended the screening at approximately 1 year interval can reduce the chance of losing contact with him/her. The availability of the mobile phone number may also reduce the cost of repeated invited calls using the telephone number at home. The third and fourth points provide an opportunity of enhancing the awareness of early detection at regular intervals.
For the effectiveness of multiple screening, in addition to fundamental short-term or longterm indicators, the brand new idea is that extra benefit achieved from the KCIS in comparison with single screening was evaluated by using Markov decision analysis (Fig. 4). Economic evaluation based on this decision framework was performed for evaluation of multiple screening opposed to single screening. The results from incremental cost-effectiveness analysis show multiple screening is more cost-effective than single screening.

Our system also makes significant contribution to epidemiological profiles, which include the elucidation of causal relationships between baseline covariates, particularly biological markers, and certain cancers or chronic diseases and the investigation of familial aggregation or genetic contribution using family-based epidemiologic design.

Compared with certain health information related to chronic disease management that only focuses on opportunistic health screening [13], our health information system for multiple screening covers an out-reach screening service. The property of out-reach service enables us to investigate epidemiological rate and distribution of five common cancers and three non-neoplastic diseases, which is very helpful to clarify the etiology of disease. The usefulness of this system in this respect is very similar to that of the computerized communityoriented primary care developed by Mettee et al. [18].

Repeated collection of biochemical variables, reproductive factors and life-style factors obtained from the KCIS program yields data for behavior risk factor surveillance, which, in turn, builds up health risk assessment for occurrence of specific diseases. Setting up a behavior risk factor surveillance system may be helpful for the improvement of primary care and secondary care regarding chronic disease as seen in a previous study addressing the measurement of clinical performance and outcomes in diabetes after the introduction of an information system [14]. This aspect should be evaluated in the future.

We also evaluated social aspects including satisfaction with the KCIS program, cue to attending the KCIS program, and engagement of community and primary care. It is important to evaluate how the KCIS program with community objectives fit in the organization of the public health or primary care system locally, i.e. for example what are the relationships with general practitioners or specialist units. Whether to offer spe- 
cialistic screening tests should be considered in relation to the need for and availability of highly specialistic treatment. This is particularly important for screening for type 2 diabetes. Those who had type 2 diabetes ascertained from the KCIS program will be referred to general practitioners for dietary, exercise and metabolic control. In addition, these subjects were also referred to an ophthalmologist to undergo screening for diabetic retinopathy.

From the aspect of data security, our system not only provides standard procedures for disease screening but also offers an environment for data exchange and referral across hospitals, which is the most important property of the health level seven (HL-7). Information systems with such communication facilities can dispense with paper delivery between the screening administration and hospitals and will increases the quality of the cancer screening. This system enables provider and client who are involved in the multiple screening program to undertake the screening activity in an efficient and systematic way.

The demand for our health information system is remarkable. It is reported that of 23 counties in Taiwan, 19 have undertaken or are in preparation for the adoption of our multiple screening model. As computerized system for these counties has been lacking or incomplete, the health information system is still run manually and by paper. The demand for the development of health information system similar to the KCIS program is urgently required.

In conclusion, a health information system for a multiple disease screening program was developed and applied to the community-based integrated screening program. Our information system incorporates several aspects that has never been addressed in computerized systems supporting single screening, including quality assurance, organization features, co-morbidity profiles, epidemiological application, social and economic consideration.

\footnotetext{
Summary points

What was already known on this topic?

Health information system plays an important role in a population-based screening for cancer and chronic disease regarding age range, inter-screening interval, development of manpower, referral and confirmatory diagnosis

Information system for single disease such as Pap smear screening has been provided but limited to infrastructure and formulation of database without extensive evaluation system
}

What this study added to our knowledge?

Health information system on multiple disease screening has been developed and applied to a community-based integrated screening regime, including five neoplastic diseases and three non-neoplastic diseases

The current health information system has been demonstrated to be a crucial factor for the development of out-reaching screening service with respect to quality assurance system, organization features, co-morbidity profiles, epidemiological applications, and social and economic considerations

\section{Acknowledgements}

We are indebted for those who were involved in this work, including Po-En Wang (Director of Keelung Health Bureau), Ting-Ting Wang (Chief of Health Prevention Division) and Health Informatics Group in Keelung Health Bureau. This project was supported by National Science Council (NSC91-2320-B010-080). This work was presented with poster section: Care of Population at AMIA symposium, 2003.

\section{References}

[1] L. Tabar, C.J. Fagerberg, A. Gad, L. Baldetorp, L.H. Holmberg, O. Grontoft, U. Ljungquist, B. Lundstrom, J.C. Manson, G. Eklund, Reduction in mortality from breast cancer after mass screening with mammography: randomised trial from the Breast Cancer Screening Working Group of the Swedish National Board of Health and Welfare, Lancet 1 (8433) (1985) 829-832.

[2] L. Nyström, I. Andersson, N. Bjurstam, J. Frisell, B. Nordenskjöld, L.E. Rutqvist, Long-term effects of mammography screening: updated overview of the Swedish randomised trials, Lancet 359 (9310) (2002) 909-919.

[3] J.S. Mandel, J.H. Bond, T.R. Church, D.C. Snover, G.M. Bradley, L.M. Schuman, F. Ederer, Reducing mortality from colorectal cancer by screening for fecal occult blood. Minnesota Colon Cancer Control Study, N. Engl. J. Med. 328 (19) (1993) 1365-1371.

[4] O. Kronborg, C. Fenger, J. Olsen, O.D. Jorgensen, O. Sondergaard, Randomised study of screening for colorectal cancer with faecal-occult-blood test, Lancet 348 (9040) (1996) 1467-1471.

[5] D.M. Eddy, Screening for cervical cancer, Ann. Intern. Med. 113 (1990) 214-226.

[6] CDC Diabetes Cost-Effectiveness Study Group, Centers for Disease Control and Prevention, Cost-effectiveness of screening for type 2 diabetes, JAMA 280 (20) (1998) $1757-1763$.

[7] T.H.H. Chen, Y.H. Chiu, D.L. Luh, M.F. Yen, H.M. Wu, L.S. Chen, T.H. Tung, C.C. Huang, C.C. Chan, M.N. Shiu, Y.P. Yeh, H.H. Liou, C.S. Liao, H.C. Lai, C.P. Chiang, H.L. Peng, C.D. Tseng, M.S. Yen, W.C. Hsu, C.H. Chen, Taiwan CommunityBased Integrated Screening Group, Community-based multiple screening model: design, implementation, and analysis of 42,387 participants, Cancer 100 (8) (2004) 1734-1743. 
[8] L.D. Marrett, S. Robles, F.D. Ashbury, B. Green, V. Goel, S. Luciani, A proposal for cervical screening information system in developing countries, Int. J. Cancer 102 (3) (2002) 293-299.

[9] L. Tabar, G. Fagerberg, T.H.H. Chen, S.W. Duffy, C.R. Smart, A. Gad, R.A. Smith, Efficacy of breast cancer screening by age. New results from the Swedish Two-County Trial, Cancer 75 (10) (1995) 2507-2517.

[10] L. Tabar, G. Faberberg, N.E. Day, L. Holmberg, What is the optimum interval between mammographic screening examinations? An analysis based on the latest results of the Swedish two-county breast cancer screening trial, Br. J. Cancer 55 (5) (1987) 547-551.

[11] C.N. Klabunde, H. Sancho-Garnier, M. Broeders, S. Thoresen, V.J. Rodrigues, R. Ballard-Barbash, Quality assurance for screening mammography data collection systems in 22 countries, Int. J. Technol. Assess. Health Care 17 (4) (2001) $528-541$.

[12] J.R. Osuch, M. Anthony, L.W. Bassett, M. DeBor, C. D’Orsi, R.E. Hendrick, M. Linver, R. Smith, A proposal for a national mammography database: content, purpose, and value, AJR Am. J. Roentgenol. 164 (6) (1995) 1329-1334.
[13] J. Cheah, Chronic disease management: a Singapore perspective, BMJ 323 (7319) (2001) 990-993.

[14] J.P. New, S. Hollis, F. Campbell, D. McDowell, E.D. Burns, T.L. Dornan, R.J. Young, Measuring clinical performance and outcomes from diabetes information systems: an observational study, Diabetologia 43 (7) (2000) 836-843.

[15] T.I. Nilsen, L.J. Vatten, Prospective study of colorectal cancer risk and physical activity, diabetes, blood glucose and BMI: exploring the hyperinsulinaemia hypothesis, Br. J. Cancer 84 (3) (2001) 417-422.

[16] F.B. Hu, J.E. Manson, S. Liu, D. Hunter, G.A. Colditz, K.B. Michels, F.E. Speizer, E. Giovannucci, Prospective study of adult onset diabetes mellitus (Type 2 ) and risk of colorectal cancer in women, J. Natl. Cancer Inst. 91 (6) (1999) $542-547$.

[17] C. La Vecchia, E. Negri, A. Decarli, S. Franceschi, Diabetes mellitus and colorectal cancer risk, Cancer Epidemiol. Biomarkers Prev. 6 (12) (1997) 1007-1010.

[18] T.M. Mettee, K.B. Martin, R.L. Williams, Tools for community-oriented primary care: a process for linking practice and community data, J. Am. Board Fam. Pract. 11 (1) (1998) 28-33.

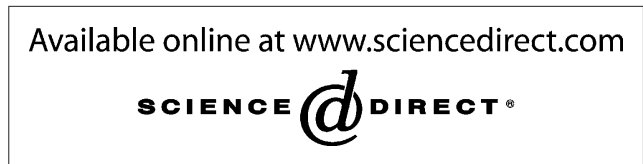

